
CÁLCULO DE LOS PARÁMETROS FUNDAMENTALES DEL SISTEMA DE LIMPIEZA DE LAS COSECHADORAS DE CEREALES.

Idalberto Macías, Benjamín Espinosa, Antonio Barrera, Lenni Ramírez, Mercedes Mayorga. 


\title{
Propuesta de metodología para el cálculo de los parámetros fundamentales del sistema de limpieza de las cosechadoras de cereales.
}

\section{Proposed methodology for the calculation of the fundamental parameters of the cereal harvesting cleaning system}

\author{
Idalberto Macías Socarrás ${ }^{1}$; Benjamín Gaskin Espinosa ${ }^{2}$; Antonio Luis Barrera Amat ${ }^{3}$; Lenni Ramírez Flores ${ }^{4}$; \\ Mercedes Arzube Mayorga ${ }^{5}$ \\ 1345 Universidad Estatal Península de Santa Elena ${ }^{2}$ Universidad de Granma. Cuba
}

\begin{abstract}
Resumen
El presente trabajo recoge, de forma resumida, una propuesta de metodología para el cálculo de los principales parámetros del sistema de limpieza de las cosechadoras de cereales; la misma para su mejor comprensión se divide en tres partes fundamentales: parámetros del sacudidor de paja, parámetros de la superficie de limpieza o tamiz y parámetros principales del ventilador. La fundamentación teórica de esta metodología, ayudará a una mejor comprensión del funcionamiento de este importante sistema para estudiantes y especialistas del tema. Consecuentemente, constituye una herramienta de trabajo para los estudiantes de las carreras de las ciencias técnicas agropecuarias, para solución de tareas relacionadas con la mecanización agropecuaria y teoría de máquinas agrícolas.
\end{abstract}

Palabras clave: Sistema de limpieza, metodología, cosechadora de cereal, eficiencia.

\begin{abstract}
The present paper summarizes a proposal for a methodology for the calculation of the main parameters of the cleaning system of grain harvesters; the same for its better understanding is divided in three fundamental parts: parameters of the straw shaker, parameters of the cleaning surface or sieve and main parameters of the fan. The theoretical basis of this methodology will help a better understanding of the functioning of this important system for students and specialists in the subject. On the other hand, it is a working tool for the students of agricultural sciences, to solve tasks related to agricultural mechanization and agricultural machinery theory.
\end{abstract}

Key word: Cleaning system, methodology, cereal harvester, efficiency. 


\section{Introducción.}

La mecanización agrícola da la oportunidad, apoyándose en los adelantos científicos y técnicos, de cumplir todos los procesos, aumentar continuamente la producción, aliviar el trabajo del hombre y elevar su productividad. Dentro de las ciencias agrónomas, esta es una de las ramas que ha obtenido en los últimos años una evolución impetuosa. García, (1989), Silveira,(1990).

El presente material ha sido elaborado con el objetivo de facilitar el trabajo a estudiantes, profesores y conocedores del tema en relación con el cálculo de los parámetros fundamentales del sistema de limpieza de las cosechadoras de cereales.

Para la elaboración de la metodología de cálculo que aquí se propone, se consultaron bibliografías de diferentes épocas y se partió, además, de una fundamentación teórica de los diferentes mecanismos y máquinas que intervienen en este proceso y de la utilización de un conjunto de disciplinas afines, tales como la Matemática, Mecánica Teórica Aplicada, Resistencia de los Materiales, Diseño de Elementos de Máquinas, Teoría de Mecanismos y Máquinas, Hidráulica y otras.

La interacción de estas disciplinas con la Teoría de Máquinas Agrícolas no se limita sólo a la aplicación de los métodos de las primeras en esta última, sino que, en reiteradas ocasiones, las tareas que han surgido en la investigación de los procesos de trabajo de las máquinas agrícolas han permitido el desarrollo de las disciplinas mencionadas. Es por ello que resulta importante su estudio. Ramos, (1989).

Por tener gran aplicación práctica, esta puede extenderse a otros centros, empresas e instituciones para que la utilicen en el diseño o perfeccionamiento de estos sistemas.

\section{Desarrollo.}

Metodología de cálculo de los parámetros fundamentales del sistema de limpieza de las cosechadoras de cereales.

Para la elaboración de esta metodología se partió de una amplia fundamentación teórica y de resultados y experiencias prácticas. La misma se divide en tres partes fundamentales: parámetros del sacudidor de paja, parámetros de las superficies de limpieza o tamiz y parámetros principales de ventilador.

\section{Parámetros del sacudidor de paja.}

Los sacudidores de paja tienen la función de separar los granos libres de la masa total de paja que proviene del aparato trillador y los dirige a los órganos cribadores, conjuntamente con la paja fina para su posterior limpieza; éste, además, conduce la masa de paja hacia el exterior de la máquina. Ochoa (1997), Valero C. (2004), Miranda, et al, (2011).

a) Ancho del sacudidor de paja (Bs), en m:

$$
\begin{gathered}
B S=(1 a 1.5) l_{b} \text { Para trilladoras de barras. (1) } \\
B S=(1.4 a 1.6) l_{b} \text { Para trilladoras de espigas (dedos) }
\end{gathered}
$$

Dónde:

$\boldsymbol{l}_{\boldsymbol{b}}$ - Longitud del cilindro trillador, en $\mathrm{m}$.

Esta longitud generalmente oscila entre 1 y 1.7 metros.

b) Longitud del sacudidor de paja ( $\boldsymbol{L s})$, en $\mathrm{m}$.

$$
L \mathbf{s}=\frac{Q \boldsymbol{s}}{\left(\mathbf{q}_{\boldsymbol{o}} \cdot \mathbf{B s} \cdot \eta_{\boldsymbol{s}}\right)}
$$

Dónde:

Qs- Productividad necesaria del sacudidor, en $\mathrm{kg} / \mathrm{s}$. $\boldsymbol{B} \boldsymbol{s}$ - Ancho del sacudidor de paja, en $\mathrm{m}$.

$\eta_{s}$ - Coeficiente de utilización del ancho del sacudidor de paja.

Según Silveira, (1990) y Ochoa, (1997) este coeficiente generalmente oscila entre 0.6 a 0.9 .

$\boldsymbol{q}_{0^{-}}$Productividad específica, en $\mathrm{kg} / \mathrm{s}-\mathrm{m}^{2}$. Para el trigo, $\mathrm{q}_{0}=1.5$ a $2.5 \mathrm{~kg} / \mathrm{s}-\mathrm{m}^{2}$, en caso de tamices bajo un régimen cinemático de $\mathrm{K}$ $=2.2$.

Para otro cultivo, la carga específica, se determina a partir de la del trigo por la siguiente expresión:

$$
q_{0}=K e \cdot q_{0}^{1}(4)
$$

Dónde:

Ke - Coeficiente de equivalencia. Tiene los siguientes valores, coincidiendo con autores como Ortega,(1993) y Miranda, et al, (2011).

$\boldsymbol{K e}=0.5$ a 0.6 , para el arroz.

$\boldsymbol{K} \boldsymbol{e}=0.6$ a 0.8, para el maíz y el chícharo.

$\boldsymbol{K} \boldsymbol{e}=0.55 \quad$ para el frijol.

$\boldsymbol{K} \boldsymbol{e}=0.1$ a 0.2 , para semillas de hierbas.

A través del cóncavo pasa hasta el $90 \%$ de los granos, entonces:

$$
Q s=Q_{M}(1-0.9 \beta)(5)
$$

Dónde:

$Q_{M}$ - Suministro medio de la masa trillada al tambor (cilíndro) trillador durante un segundo. 


$$
Q_{M}=\frac{\left(Q c \cdot B \cdot V m \cdot 10^{4}\right)}{\beta}(6)
$$

Dónde:

$\boldsymbol{Q c}$ - Rendimiento de la cosecha, en $\mathrm{kg} \cdot \mathrm{ha}^{-1}$.

$\boldsymbol{B}$ - Ancho de corte de la combinada, en $\mathrm{m} / \mathrm{s}$.

$\boldsymbol{V} \boldsymbol{m}$-Velocidad de la combinada cosechando, en $\mathrm{m} / \mathrm{s}$.

$\boldsymbol{\beta}$ - Relación entre la cantidad total de granos y toda la masa suministrada durante la trilla. Generalmente oscila entre $\boldsymbol{\beta}=0.3$ a 0.5 .

c) Velocidad de giro del sacudidor de paja ( $\varpi)$, en r/s.

$$
\varpi=\sqrt{\frac{K \cdot g}{r}}
$$

Dónde:

$\boldsymbol{K}$ - Coeficiente de régimen cinemático. Oscila entre 2 y 3 , se recomienda:

$\boldsymbol{K}=2.2$ a 2.6, para sacudidores de paja con 4 zarandas.

$\boldsymbol{K}=2.4$ a 2.8, para sacudidores de paja con 5 zarandas.

$\boldsymbol{K}=2.7$ a 3, para sacudidores de paja con 6 zarandas. $\boldsymbol{r}$ - Radio de la manivela del árbol del sacudidor de paja, en $\mathrm{m} . \mathrm{r}=(45$ o 50$) 10^{-3} \mathrm{~m}$.

$\boldsymbol{g}$ - Aceleración de la gravedad, en $\mathrm{m} / \mathrm{s}^{2}$.

Expresado en r/min.:

$$
\boldsymbol{n}=\frac{\boldsymbol{3 0}}{\boldsymbol{\pi}} \sqrt{\frac{\boldsymbol{K} \cdot \boldsymbol{g}}{\boldsymbol{r}}}(8)
$$

\section{Parámetros de la superficie de limpieza o tamiz.}

La clasificación mecánica se efectúa con un movimiento relativo del material y de la superficie de trabajo de la criba. Como resultado se obtienen dos productos: granos que pasaron a través del tamiz y paja u otro tipo de materia extraña (tierra y otras semillas)que quedaron en la parte superior del tamiz. El trabajo de las cribas se valora por dos índices: por la efectividad de la clasificación y por la productividad de las cribas.Ochoa, R. (1997), Valero C. (2007).

Las superficies planas de separación sometidas a un movimiento vibratorio, se utilizan para la separación de partículas de paja y otras impurezas de los granos, así como, para la separación de los granos según sus características dimensionales y peso específico. Las vibraciones de las superficies provocan el desplazamiento del material por ésta y, a su vez, este movimiento debe de ser tal que produzca una separación óptima. Ortiz-Cañavate, J (2005), Miranda A, et al, (2011). a) Área de la superficie de limpieza o tamiz $\left(\boldsymbol{F}_{T}\right)$, en $\mathrm{m}^{2}$ :

$$
\boldsymbol{F}_{\boldsymbol{T}}=\frac{\boldsymbol{Q}_{\boldsymbol{T}}}{\boldsymbol{q}_{o}}
$$

Dónde:

$\boldsymbol{Q}_{\boldsymbol{T}}$ - Cantidad de material trillado o suministrado para la limpieza en un segundo, en $\mathrm{kg} . \mathrm{s}^{-1}$.

$$
\boldsymbol{Q}_{\boldsymbol{T}}=\frac{\boldsymbol{\beta} \cdot \boldsymbol{Q}_{\boldsymbol{M}}(10)}{\boldsymbol{\beta}_{\boldsymbol{g}}}
$$

Dónde:

$\boldsymbol{\beta}_{\boldsymbol{g}}$ - Relación entre la cantidad total de granos y la masa cosechada que se suministra para la limpieza. $\boldsymbol{\beta}_{g}=0.75$ a 0.9 .

b) Ancho de limpieza $\left(\boldsymbol{B}_{T}\right)$, en m:

$$
\boldsymbol{B}_{T}=0.9 \mathrm{Bs}
$$

c) Largo de la limpieza $\left(\boldsymbol{L}_{T}\right)$, en m:

$$
\boldsymbol{L}_{\boldsymbol{T}}=\frac{\boldsymbol{F}_{\boldsymbol{T}}}{\boldsymbol{B}_{\boldsymbol{T}}}
$$

\section{Parámetros principales del ventilador.}

El ventilador es una máquina que desplaza el medio gaseoso con un grado de elevación de presión de hasta 1.15. Los ventiladores que se utilizan en las máquinas agrícolas, por lo general son especiales, de doble succión, centrífugos de paletas rectas con un número que oscila entre cuatro y seis, y de presión media y baja. Mataix, (1975); Chercasski, (1986), Ramos, (1989).

a) Flujo de aire necesario para la limpieza $(\boldsymbol{Q a})$, en $\mathrm{m}^{3} \cdot \mathrm{s}^{-1}$.

$$
\mathbf{Q a}=\lambda \cdot \boldsymbol{\varepsilon} \cdot \boldsymbol{Q}_{\boldsymbol{M}}(13)
$$

Dónde:

$\lambda$ - Coeficiente que caracteriza la concentración del medio.

$\boldsymbol{\varepsilon}$ - Relación entre las impurezas a eliminar y toda la masa en proceso de limpieza.

Estos valores dependen de la productividad del grano y de la cantidad de residuos, los mismos varían entre $\lambda=0.014$ a 0.039 y $\varepsilon=10$ a 16 .

b) Velocidad del chorro de aire en la ventana de salida del ventilador $(\boldsymbol{V a})$, en $\mathrm{m} / \mathrm{s}$ :

$$
\boldsymbol{V a}=\alpha_{\mathbf{a}} \cdot \operatorname{Vcr}(14)
$$

Dónde: 
$\alpha_{a}$ - Coeficiente que caracteriza la velocidad crítica del aire o coeficiente de exceso de aire. Se recomienda $\alpha_{a}=1.5$ a 3 .

Vcr - Velocidad crítica de las impurezas, en $\mathrm{m} / \mathrm{s}$. Los valores de Vcroscilan entre los 4 y 6 m/s.

c) Presión total del aire suministrado por el ventilador $(\Delta p)$, en $\mathrm{Pa}$.

$$
\Delta p=\frac{\rho \cdot \operatorname{Vas}^{2}}{2}
$$

Dónde:

$\boldsymbol{\rho}$ - Densidad del aire, en $\mathrm{kg} / \mathrm{m}^{3}$. En Cuba, el valor de la densidad del aire a presión atmosférica y temperatura de $28^{\circ} \mathrm{C}$ es de $1.174 \mathrm{~kg} / \mathrm{m}^{3}$.

d) Carga real ( $\left.\boldsymbol{H}_{\text {real }}\right)$, en $\mathrm{m}$.

$$
\boldsymbol{H}_{\text {real }}=\frac{\Delta p}{\rho \cdot g}(16)
$$

e) Carga teórica $\left(\boldsymbol{H}_{T}\right)$, en $\mathrm{m}$.

$$
\boldsymbol{H}_{\boldsymbol{T}}=\frac{\boldsymbol{H}_{\text {real }}}{\boldsymbol{\eta}_{\boldsymbol{h}}}
$$

Dónde:

$\eta_{\boldsymbol{h}}$ - Eficiencia hidráulica. Para los ventiladores de estas máquinas oscila entre el 30 y el 50 $\%$.

f) Diámetro de absorción del ventilador (Da), en m.

$$
D a \sqrt{\frac{2 \cdot Q a}{\pi \cdot V a a}}(18)
$$

Dónde:

$\boldsymbol{V} \boldsymbol{a} \boldsymbol{a}$ - Velocidad del aire en la ventana de absorción, en $\mathrm{m} / \mathrm{s}$. $(\boldsymbol{V a a} \approx \boldsymbol{V a})$.

g) Diámetro interior $\left(\boldsymbol{D}_{1}\right)$ y exterior $\left(\boldsymbol{D}_{2}\right)$ de las aspas del ventilador, en $\mathrm{m}$.

$$
\begin{aligned}
& D_{1}=0.95 \mathrm{Da} . \\
& D_{2}=1.2 \mathrm{Da} .
\end{aligned}
$$

h) Altura de la ventana de salida (a) del ventilador, en $\mathrm{m}$.

$$
a=(0.4 a \text { 0.6) Da. (21) }
$$

i) Ancho de la ventana de salida (b) del ventilador, en $\mathrm{m}$.

$$
\boldsymbol{b}=\boldsymbol{B}_{\boldsymbol{T}}
$$

j) Potencia útil necesaria para el trabajo del ventilador $(\boldsymbol{N})$, en $\mathrm{kW}$.

$$
N=Q a \cdot \rho \cdot H_{T} \cdot g \cdot 10^{-3}(23)
$$

\section{Conclusiones}

1. La metodología para el cálculo de los principales parámetros constructivos del sistema de limpieza de las cosechadoras de cereales fue elaborada partiendo de una amplia fundamentación teórica de los diferentes mecanismos y máquinas que intervienen en este proceso y de la utilización de un amplio conjunto de disciplinas afines.

2. Los resultados de cálculos obtenidos con el empleo de esta metodología se corresponden con los que presentan las máquinas, comprobándose su validez.

3. La metodología confeccionada tiene aplicación práctica y puede extenderse a centros de enseñanzas, empresas e instituciones para que la utilicen en el diseño o perfeccionamiento de estos sistemas.

\section{Bibliografía.}

[1] Chercasski, M. (1986). Bombas Ventiladores Compresores. Moscú: Editorial Mir.

[2]García, A. (1989). Cosechadoras de Cereales: Cosechadoras de Granos y Semillas. Italia. Editora de la oficina regional de la FAO para América Latina y el Caribe. Roma.

[3]JOHN DEERE. (1990). Cuadro comparativo de combinadas JOHN DEERE. Maquinaria de Humaya S.A. de C.V. Monterrey: Industrias JOHN DEERE S.A. de C.V. Monterrey N. L.

[4]JOHN DEERE. (1990). Fórmulas para medir la capacidad de una combinada. Maquinaria de Humaya S.A. Industrias JOHN DEERE S.A. de C.V. México. Monterrey N.L.

[5]JOHN DEERE. (1990). Getting maximum productivity withyour 9000 Series Combine. U.S.A: JOHN DEERE S.A.

[6]Mataix, C. (1975). Turbomáquinas Hidráulicas. Madrid: Editora ICAI.

[7]Ochoa, R. (1997). Metodología de cálculo de los parámetros fundamentales del sistema de limpieza de las cosechadoras de cereales Tesis Presentada en Opción al Título de Master en Maquinaria Agrícola. Universidad de Holguín. 
[8]Ortega, L. (1993). Manual de Explotaciones Agrícolas. Madrid: Ediciones Mundi-Prensa.

[9]Ortiz-Cañavate, J.(2005). Las Máquinas Agrícolas y su Aplicación. Editorial MundiPrensa. 7 ma edición.

[10]Ramos, N. (1989). Bombas Ventiladores Compresores. Ciudad de la Habana: Editorial I.S.J.A.E.

[11]Shubin, S. y Pedre, C. (1982). Diseño de Máquina Industrial. Ciudad de la Habana: Editorial Pueblo y Educación.

[12]Silveira, J. (1990). Máquinas Agrícolas. Ciudad de la Habana: Editorial Pueblo y Educación.
[13]Silveira, J. (1982). Teoría y Cálculo de Máquinas Agrícolas. Ciudad de la Habana: Editorial Pueblo y Educación.

[14] Valero C. (2007). Cosechadoras de cereal: historia, elementos y funcionamiento. Departamento de Ingeniería Rural UPM. Salvado de: http://oa.upm.es/6375/1/Valero_71.pdf

[15] Valero C. (2004). Sistemas de trilla y separación en cosechadoras de cereal. Departamento de Ingeniería Rural UPM. Salvado de: http://oa.upm.es/6311/1/Valero_48.pdf

[16]Miranda A, et al, (2011). Determinación del contenido de impurezas durante la cosecha de arroz. Revista Ciencias Técnicas Agropecuarias vol.20 no.4. ISSN: 2071-0054. 